\title{
Neutrophil to lymphocyte ratio and platelet to lymphocyte ratio as a risk factor for mortality in peruvian adults with chronic kidney disease.
}

\author{
Gianfranco Umeres-Francia1 ${ }^{1}$, María Rojas-Fernández ${ }^{1}$, Percy Herrera Añazco ${ }^{2}$, and \\ Vicente Benites-Zapata ${ }^{3}$ \\ ${ }^{1}$ Universidad Peruana de Ciencias Aplicadas \\ ${ }^{2}$ Hospital Nacional 2 de mayo \\ ${ }^{3}$ Universidad San Ignacio de Loyola
}

February 2, 2021

\begin{abstract}
Objective: To assess the association between NLR and PLR with all-cause mortality in Peruvian patients with CKD Methods: We conducted a retrospective cohort study in adults with CKD in stages 1 to 5 . The outcome variable was mortality and as variables of exposure to NLR and PLR. Both ratios were categorized as high with a cut-off point of 3.5 and 232.5 ; respectively. We carried out a Cox regression model and calculated crude and adjusted hazard ratios (HR) with their $95 \%$ confidence interval (95\%CI). Results: We analyzed 343 participants with a median follow-up time of 2.45 years (2.08-3.08). The frequency of deaths was $17.5 \%(\mathrm{n}=60)$. In the crude analysis, the high NLR and PLR were significantly associated with all-cause mortality $(\mathrm{HR}=2.01 ; 95 \% \mathrm{CI}: 1.11-3.66)$ and $(\mathrm{HR}=2.58 ; 95 \% \mathrm{CI}: 1.31-5.20)$. In the multivariate model, after adjusting for age, sex, serum creatinine, CKD stage, albumin and hemoglobin, the high NLR and PLR remained as an independent risk factor for all-cause mortality, $(\mathrm{HR}=2.10 ; 95 \% \mathrm{CI}: 1.11-3.95)$ and ( $\mathrm{HR}=2.71 ; 95 \% \mathrm{CI}: 1.28-5.72)$. Conclusion: Our study suggests the relationship between high NLR and PLR with all-cause mortality in patients with CKD.
\end{abstract}

Neutrophil to lymphocyte ratio and platelet to lymphocyte ratio as a risk factor for mortality in peruvian adults with chronic kidney disease.

Gianfranco Eddú Umeres-Francia ${ }^{1}$, María Valentina Rojas-Fernández ${ }^{1}$, Percy Herrera-Añazco ${ }^{1}$, Vicente Aleixandre Benites-Zapata ${ }^{2}$

${ }^{1}$ Universidad Peruana de Ciencias Aplicadas, School of Medicine, Lima, Peru

${ }^{2}$ Universidad San Ignacio de Loyola, Unidad de Investigación para la Generación y Síntesis de Evidencias en Salud, Lima, Peru.

\section{Corresponding author:}

Vicente A. Benites-Zapata

vbeniteszapata@gmail.com

Avenida La Fontana 750, La Molina, Lima, Peru;

51995523081

Disclosure statement: None to declare. 
Authors' contributions: GEUF, MVRF, PHA and VABZ have participated in the conception of the article, the data collection, its writing and approval of the final version. In addition, VABZ performed the data analysis.

Funding sources: This research did not receive any specific grant from funding agencies in the public, commercial or not-for-profit sectors.

Abstract.

Objective: To assess the association between NLR and PLR with all-cause mortality in Peruvian patients with CKD

Methods: We conducted a retrospective cohort study in adults with CKD in stages 1 to 5 . The outcome variable was mortality and as variables of exposure to NLR and PLR. Both ratios were categorized as high with a cut-off point of 3.5 and 232.5; respectively. We carried out a Cox regression model and calculated crude and adjusted hazard ratios (HR) with their $95 \%$ confidence interval $(95 \% \mathrm{CI})$.

Results: We analyzed 343 participants with a median follow-up time of 2.45 years (2.08-3.08). The frequency of deaths was $17.5 \%(\mathrm{n}=60)$. In the crude analysis, the high NLR and PLR were significantly associated with all-cause mortality $(\mathrm{HR}=2.01 ; 95 \% \mathrm{CI}: 1.11-3.66)$ and $(\mathrm{HR}=2.58 ; 95 \% \mathrm{CI}: 1.31-5.20)$. In the multivariate model, after adjusting for age, sex, serum creatinine, CKD stage, albumin and hemoglobin, the high NLR and PLR remained as an independent risk factor for all-cause mortality, (HR=2.10; 95\% CI:1.11-3.95) and $(\mathrm{HR}=2.71 ; 95 \%$ CI:1.28-5.72).

Conclusion: Our study suggests the relationship between high NLR and PLR with all-cause mortality in patients with CKD.

Keywords: Kidney Failure, Chronic; Neutrophils; Blood Platelets; Lymphocytes; Mortality

\section{What's knows}

Background: The neutrophil-to-lymphocyte ratio (NLR) and platelet-to-lymphocyte ratio (PLR) may be prognostic markers in chronic illness

\section{What's new}

After adjusting for age, sex, serum creatinine, chronic kidney disease stage, albumin and hemoglobin, the high NLR and PLR remained as an independent risk factor for all-cause mortality in chronic kidney disease patients

\section{Introduction}

Chronic kidney disease (CKD) is defined as the presence as abnormalities of kidney structure or function, present for more than three months, with implications for health(1). Currently, stakeholders recognized CKD as a severe public health problem related to deterioration in the quality of life and high costs for the health system(2). Patients with CKD have a lower life expectancy and a higher rate of cardiovascular complications, as well as anemia, bone disease, infections and cancer(3). According to Global Burden Disease, Globally, in 2017, 1.2 million people died from CKD, and the global all-age mortality rate from CKD increased $41.5 \%$ between 1990 and 2017(4). In Peru, for the period 2010-2016, the prevalence of CKD increased by 300\%, and although rate the standardized mortality decreased by $10 \%$ it is still high for a country with limited economic resources(5). Due to the high burden of the disease, it is essential to know the factors associated with higher mortality.

Although there are different factors associated with mortality in patients with CKD, there is interest in searching for new markers. The neutrophil to lymphocyte ratio (NLR) and platelet to lymphocyte ratio (PLR) are markers of chronic inflammation. These markers are obtained easily from the quotient between neutrophil and lymphocyte or platelet and lymphocyte blood counts; respectively(6-8). Neutrophils are effector cells of the immune system, which rise in inflammation and generate an increase in oxidative stress(9). Similarly, 
platelets have immunomodulatory properties, which lead to inflammation, thrombosis and cancer $(10,11)$. Indeed, the contribution of interactions between neutrophils and platelets in infections and inflammatory processes have begun to be intensively studied $(12,13)$.

Specific lymphocyte subtypes, such as Th2 and regulatory $\mathrm{T}$, decrease inflammation by different routes, such as GATA3 and FOXP3(14). Therefore, the elevation of the NLR and PLR reflects a pro-inflammatory imbalance, as the effector cells are increased, and the regulatory cells are decreased. In CKD, the oxidative stress, increase in pro-inflammatory cytokines (PCR, IL-6, TNF-a) and the poor nutritional status caused by this imbalance contribute to the progression of disease(15).

The NLR and PLR have proven to be an marker of mortality and complications in pathologies such as heart failure, peptic ulcer perforation, chronic obstructive pulmonary disease, rheumatic diseases, neoplasms, and others(16-18). In patients with CKD, the NLR and PLR predict disease progression and the presence of albuminuria(19,20). Although several studies have suggested that both markers also predict mortality in patients with CKD, there are contradictory results(21), they are studies done in dialysis patients $(22,23)$, or patients with CKD in stages 1-5, but in Asian population(24). Because mortality and associated factors differ according to the stage of the disease and in Hispanic population(25-27), this study aims to assess the association between NLR and PLR with all-cause mortality in Peruvian patients with CKD who was attending tertiary hospital.

\section{Materials and methods}

\section{Population and study design:}

We conducted a retrospective, analytical and observational cohort study in a population consisted in patients with chronic kidney disease (CKD) with stages 1- 5 from the Hospital Edgardo Rebagliati Martins (EsSalud) in Lima, Peru. The inclusion criteria were: Patient with CKD who enters the surveillance system of the Chronic Kidney Disease Management Unit (SSCKD) between 2016 and 2018 and patient with complete blood count with a maximum separation month between the start of follow-up and the blood test. The exclusion criteria were: Patient with CKD stage 5 in dialysis and patient with a history of renal transplantation.

\section{Sample and statistical power calculation:}

We conducted a census for the collection of participants in the established study period. The total sample of our study was 343 participants. For the statistical power calculation, we used the study by Tatar et al., who evaluated the association of high NLR and mortality in elderly patients with CKD stages 3-5. In this study, the survival reported in the Kaplan-Meier Curves at sixty months in the group with increased NLR was $50.5 \%$, while in the group with normal NLR, it was $85.6 \%$. We carried out the calculations using a $95 \%$ confidence interval (CI) and obtained a statistical power of $99 \%$

\section{Variables}

The exposure variables were the neutrophil/lymphocyte ratio (NLR) and the platelet/lymphocyte ratio (PLR), defined as the quotient between neutrophil or platelet count and lymphocyte count. We calculated both ratios using the patient's complete blood count, and defined elevates NLR and PLR; as a cut-off point of 3.5 and 232.5; respectively(14). The primary outcome was mortality, which is a dependent variable composed of the probability of dying and the time until the development of the event. Other variables of interest included age (years), sex, glomerular filtration rate (GFR) (ml $/ \mathrm{min} / 1.73 \mathrm{~m} 2)$, CKD stage (stratified into three groups: initial stages ( 1 and 2), intermediate stages (3a and $3 \mathrm{~b}$ ) and advanced stages (4 and 5), platelets. $(\mathrm{K} / \mathrm{ul})$, albumin $(\mathrm{g} / \mathrm{dl})$, hemoglobin $(\mathrm{g} / \mathrm{dl})$ and comorbidities such as diabetes and hypertension.

\section{Data collection procedure:}

To collect the data, we obtained a list of patients who entered the SSCKD during the 2016-2018 period. The SSCKD was created from patients referred from primary care centers attached to the hospital. In the healthcare centers, the physician performed the CKD screening estimating the GFR using the modification dialysis renal disease 4 (MDRD4) formula and the albumin/creatinine ratio in urine at random. Physicians 
referred patients with an estimated GFR $<60 \mathrm{~mL} / \mathrm{min} / 1.73 \mathrm{~m} 2$ for care with the nephrologist. In the first visit, the nephrologist repeated creatinine and other laboratory data were requested.

Subsequently, we reviewed the laboratory results requested by the nephrologist for the patient's admission to the database, using the software called "RESULAB". We collected serum creatinine $(\mathrm{mg} / \mathrm{dl})$, eGFR $(\mathrm{ml} / \mathrm{min} / 1,73 \mathrm{~m} 2)$, glucose $(\mathrm{mg} / \mathrm{dl})$, total protein $(\mathrm{g} / \mathrm{dL})$, albumin $(\mathrm{g} / \mathrm{dL})$, glutamicpyruvic transaminases $(\mathrm{U} / \mathrm{L})$, glutamic-oxaloacetic transaminases $(\mathrm{U} / \mathrm{L})$, total bilirubin $(\mathrm{mg} / \mathrm{dL})$, alkaline phosphatase $(\mathrm{U} / \mathrm{L})$, globulin $(\mathrm{g} / \mathrm{dL})$, hemoglobin $(\mathrm{g} / \mathrm{dl})$, platelets $(\mathrm{K} / \mathrm{uL})$, neutrophils $(\mathrm{K} / \mathrm{uL})$ and lymphocytes $(\mathrm{K} / \mathrm{uL})$. We determined the vital status and date of death through the consultation of the National Registry of Identification and Civil Status (RENIEC, in Spanish) portal. (https://portaladminusuarios.reniec.gob.pe/validacionweb/index.html\#no-back-button).

For non-survivors patients, we collected the date of death for any cause to establish the time until the event. For those patients who did not die during the established period, the closure of the follow-up was Oct 31, 2019. We marked the beginning of the follow-up by the patient's admission to the SSCKD.

\section{Statistical Analysis}

We summarize measures for the numerical and categorical variables. If the distribution of the data was symmetric, we showed the variables as means with standard deviation or, failing that, as medians with interquartile range.

We applied the Student's T-test to compare numerical variables with symmetrical distributions. If the distributions were asymmetrical, we used the Mann-Whitney U-test. We used the Kaplan-Meier method and the log-rank test to compare survival functions. To contrast the study hypothesis, we calculated the crude and adjusted Cox regression model or the proportional hazards model. We calculated crude and adjusted Hazard Ratio (HR), with a 95\% confidence interval (CI). We entered the confounding variables into the adjusted model if they had $\mathrm{p}<0.05$ in the crude analysis. Finally, we verified compliance with the assumption of hazard proportionality in the Cox regression model. All analyses were performed with the STATA statistical package version 14.0.

Ethical approval: All procedures performed in studies involving human participants were in accordance with the ethical standards of the institutional and/or national research committee at which the studies were conducted (Universidad Peruana de Ciencias Aplicadas; PI130-19)

\section{Results}

We reviewed 356 clinical records from patients with CKD who entered SSCKD between 2016 and 2018. We excluded 12 patients because they did not have complete laboratory tests and one who did not present a death date. Finally, we included 343 patients in the analysis. The mean age was $78.3( \pm 11.9)$ years, and the median follow-up time was 2.45 years (2.08-3.08). In the population studied, the predominant gender was male, with $62.9 \%(\mathrm{n}=216)$. Regarding comorbidities, $64.7 \%$ were found to have hypertension, while $30 \%$ have diabetes mellitus. On the other hand, the average of eGFR was $47.4( \pm 10.5) \mathrm{ml} / \mathrm{min} / 1.73 \mathrm{~m}^{2}$ and the ERC stage included $22(6.4 \%)$ patients in stages 1-2, $311(90,7 \%)$ patients in stages 3a-3b and $10(2.9 \%)$ patients in stages 4-5. Demographic and laboratory characteristics are shown in Table 1.

Regarding the exposure's variables, 14.6\% $(\mathrm{n}=50)$ had high NLR and $8.2 \%(\mathrm{n}=28)$ high PLR. In total, $17.5 \%(\mathrm{n}=60)$ died at the end of follow-up. Participants with elevated NLR had higher serum levels of alkaline phosphatase (114 vs $100 \mathrm{U} / \mathrm{L} ; \mathrm{p}=0.03$ ) and platelets (260 vs $234 \mathrm{~K} / \mathrm{uL} ; \mathrm{p}<0.01$ ) than participants with normal NLR (Table 1). Participants with elevated PLR had a lower average age (72.6 vs 78.8 years; $\mathrm{p}<0.01$ ), glutamic oxaloacetic transaminase (19 vs $23 \mathrm{U} / \mathrm{L} ; \mathrm{p}=0.02)$ and hemoglobin (11.2 vs $12.2 \mathrm{~g} / \mathrm{dL}$; $\mathrm{p}<0.01)$ compared to participants with normal PLR. In contrast, participants with elevated PLR had higher serum levels of neutrophils ( 4.5 vs $3.9 \mathrm{~K} / \mathrm{uL} ; \mathrm{p}=0.03$ ) (Table 2 ).

The mortality of patients with high NLR was $28 \%$ compared to $15.7 \%$ of the group with normal NLR ( $\mathrm{p}=$ 0.03). Likewise, mortality was $35.7 \%$ in those with high PLR and $15.6 \%$ in those with normal PLR ( $\mathrm{p}=0.01)$. 
The mortality rate in the group with high NLR was 12.5 deaths per 100 person-years of follow-up, while in the group with high PLR, it was 16 deaths per 100 person-years of follow-up. Kaplan-Meier curves showed a statistically significantly worse survival function for participants with high NLR and PLR at the end of follow-up (Figure 1 and 2).

Mean age was higher in the group of the dead (81.9 vs 77.5 years; $\mathrm{p}<0.01)$ and there was more mortality in males $(21.3$ vs $11 ; \mathrm{p}=0.01)$. The most advanced stages of chronic kidney disease had higher mortality, $9.1 \%$, $16.4 \%$ and $70 \%$, respectively. In the laboratory profile, creatinine levels were higher in the dead group (1.4 vs $1.6 \mathrm{mg} / \mathrm{dL} ; \mathrm{p}<0.01)$. On the other hand, both hemoglobin (11.7 vs. $12.3 \mathrm{~g} / \mathrm{dL} ; \mathrm{p}=0.03$ ) and albumin (4.0 vs. $4.2 \mathrm{mg} / \mathrm{dL} ; \mathrm{p}<0.01$ ) had lower values in the dead group (Table 3 ).

In the crude analysis, the high NLR was significantly associated with all-cause mortality (HR $=2.01 ; 95 \%$ CI: 1.11-3.66). Likewise, the high PLR was significantly associated with all-cause mortality (HR $=2.58 ; 95 \%$ CI: 1.31-5.20) (Table 4).

In the multivariate model, after adjusting for age, sex, serum creatinine, chronic kidney disease stage, albumin and hemoglobin, the high NLR and PLR remained as an independent risk factor for all-cause mortality, (HR $=2.10 ; 95 \%$ CI: $1.11-3.95)$ and $(\mathrm{HR}=2.71 ; 95 \%$ CI: 1.28-5.72); respectively (Table 4).

\section{Discussion.}

The present study suggests the relationship between high NLR and PLR with all-cause mortality in patients with CKD. Thus, those patients who had a high NLR or PLR showed twice the risk of dying compared to those who had these ratios within normal limits. Our results are similar to a study conducted in 350 Japanese patients with CKD in stages 1 to 4, in which a high NLR was associated with a higher probability of requiring dialysis and death(15). Likewise, they are similar to a study done in 165 Turkish patients with CKD in stages 1 to 3 that found that a high NLR was associated with higher mortality from all causes(21). In contrast, Chinese patients with CKD in stages 1 to 4, did not find an association between NLR and cardiovascular mortality or of any cause(20). Methodological differences can explain these discrepancies. Although the study included a more significant number of patients (938) and was multicenter, it also had a smaller number of diabetic patients $(23.8 \%)$ compared to the Japanese (37\%), Turkish $(41.4 \%)$ or in our study (30\%), and could explain these differences, due to the higher mortality in diabetic patients(28). Similarly, the mean age of the Chinese patients was comparatively lower (52.8) than that of the patients in the Japanese (68), Turkish (73.8) and our studies (78.3), which is essential because older age is associated with higher mortality in $\operatorname{CKD}(29)$.

The association of NLR and survival is justified in that is a marker of inflammation that predicts complications and mortality in patients with chronic diseases. This biomarker indicates an imbalance between effector cells (neutrophils), which reflect oxidative stress and regulatory cells (lymphocytes), which decrease the pro-inflammatory state(30). This relationship between neutrophil count and lymphocyte count is a new measure of inflammation in different diseases with systemic inflammation such as $\operatorname{CKD}(31)$ Uncontrolled inflammation causes glomerular, tubular and interstitial damage that leads to renal hemodynamic imbalance and failure to control blood pressure(30).

Regarding PLR and mortality, we have similar findings to a study in patients with rapidly progressive glomerulonephritis in which both NLR and PRL were associated with mortality(32). However, a Turkish study no found association between PRL and mortality or the need of dialysis, but, the PRL group that died was a mean of $112 \pm 267$, while in our study a high PLR was considered as cut-off $>232.5(21)$. In that sense, another study found that relationship between PRL and mortality is not a linear relationship and that it can follow a curve in J-shaped that differs according to ethnicity (PLR[?]83.18) and albumin (PLR [?] 113.89)(42). It is likely that, as with NLR, systemic inflammation may play a fundamental role. Increase in platelets or an increase in lymphocytes is typical to find in patients with inflammatory states(33). Platelet release can be modulated by inflammatory cytokines as a mechanism for rapid recovery, while decreased lymphocytes may be due to increased lymphocyte apoptosis and suppressed lymphocyte production caused by high levels of cortisol in response to inflammation $(33,34)$. 
Although the association between NLR, PLR and mortality may be related to more significant inflammation, it would also be mediated by CKD progression, because in the advanced stages of the disease, there is higher mortality (34). However, after sensitivity analysis, removing stage 4 and 5 participants, lymphocyte ratios remained as an independent risk marker for mortality. This finding could suppose other additional routes to the progression of the disease to explain this association in our population. Moreover, several studies reported NLR and PLR associated with albuminuria (regardless of GFR) and decrease in GFR.(19,35). So, this finding becomes relevant due to the association between albuminuria, CKD progression, and mortality $(32,33)$.

Our study has limitations. First, being a retrospective study, the data obtained could have deficiencies in its registration. Second, there is the possible inclusion of patients with subclinical inflammatory and infectious diseases, which are not registered in the SSCKD or RESULAB database. Third, no other possible factors that could potentially influence mortality, such as nutritional status, albuminuria, cardiovascular disease, obesity, smoking, and acute renal injury, have been evaluated. Fourth, by the design of our study, we can only assume that lymphocyte ratios are biomarkers of risk and not of causality. Finally, the measurement of the ratios has only been carried out at the beginning of the follow-up; therefore, we not evaluated variations in time and influence in the mortality.

In conclusion, our study suggests the relationship between high NLR and PLR with all-cause mortality in patients with CKD. The patients who had a high NLR or PLR showed twice the risk of dying compared to those who had these ratios within normal limits. These ratios are constructed with values usually collected in hemograms commonly used for patient follow-up. Being constructed based on a cheap and common exam and, due to our results, we believe that it could be considered within the usual practice in resource countries limited.

\section{References}

1. Levin A, Stevens PE, Bilous RW, Coresh J, De Francisco ALM, De Jong PE, et al. Kidney disease: Improving global outcomes (KDIGO) CKD work group. KDIGO 2012 clinical practice guideline for the evaluation and management of chronic kidney disease, Kidney International Supplements. 2013;3(1):1-150.

2. Levin A, Tonelli M, Bonventre J, Coresh J, Donner JA, Fogo AB, et al. Global kidney health 2017 and beyond: a roadmap for closing gaps in care, research, and policy. Lancet. 2017;390(10105):1888-1917.

3. Webster AC, Nagler E V., Morton RL, Masson P. Chronic Kidney Disease. Lancet.2017; 389(10075):12381252

4. Bikbov B, Purcell CA, Levey AS, Smith M, Abdoli A, Abebe M, et al. Global, regional, and national burden of chronic kidney disease, 1990-2017: a systematic analysis for the Global Burden of Disease Study 2017. Lancet. 2020;395(10225):709-733.

5. Atamari-Anahui N, Ccorahua-Rios MS, Condori-Huaraka M, Huamanvilca-Yepez Y, Amaya E, HerreraAnazco P. Epidemiology of chronic kidney disease in Peru and its relation to social determinants of health. Int Health. 2020;12(4):264-271.

6. Fei Y, Wang X, Zhang H, Huang M, Chen X, Zhang C. Reference intervals of systemic immuneinflammation index, neutrophil to lymphocyte ratio, platelet to lymphocyte ratio, mean platelet volume to platelet ratio, mean platelet volume and red blood cell distribution width-standard deviation in healthy Han adults in Wuhan region in central China. Scand J Clin Lab Invest. 2020;80(6):1-8.

7. Gasparyan AY, Ayvazyan L, Mukanova U, Yessirkepov M, Kitas GD. The platelet-to-lymphocyte ratio as an inflammatory marker in rheumatic diseases. Ann Lab Med. 2019;39(4):345-357.

8. Kurtul A, Ornek E. Platelet to Lymphocyte Ratio in Cardiovascular Diseases: A Systematic Review. Angiology. 2019; 70(9):802-818.

9. Kotani K, Sakane N. White blood cells, neutrophils, and reactive oxygen 4metabolites among asymptomatic subjects. Int J Prev Med. 2012;3(6):428-431. 
10. Nicolai L, Schiefelbein K, Lipsky S, Leunig A, Hoffknecht M, Pekayvaz K, et al. Vascular surveillance by haptotactic blood platelets in inflammation and infection. Nat Commun. 2020;11(1):5778.

11. Saito R, Shoda K, Maruyama S, Yamamoto A, Takiguchi K, Furuya S, et al. Platelets enhance malignant behaviours of gastric cancer cells via direct contacts. Br J Cancer. 2020; doi: 10.1038/s41416-020-01134-7.

12. Portier I, Campbell RA. Role of Platelets in Detection and Regulation of Infection. Arterioscler Thromb Vasc Biol. 2020;ATVBAHA120314645.

13. Warren E, Teskey G, Venketaraman V. Effector Mechanisms of Neutrophils within the Innate Immune System in Response to Mycobacterium tuberculosis Infection. J Clin Med. 2017;6(2):15.

14. Valga F, Monzon T, Henriquez F, Anton-Perez G. Neutrophil-to-lymphocyte and platelet-to-lymphocyte ratios as biological markers of interest in kidney disease. Nefrologia. 2019;39(3):243-249.

15. Yoshitomi R, Nakayama M, Sakoh T, Fukui A, Katafuchi E, Seki M, et al. High neutrophil/lymphocyte ratio is associated with poor renal outcomes in Japanese patients with chronic kidney disease. Ren Fail. 2019;41(1):238-243.

16. Seropian IM, Romeo FJ, Pizarro R, Vulcano NO, Posatini RA, Marenchino RG, et al. Neutrophil-tolymphocyte ratio and platelet-to-lymphocyte ratio as predictors of survival after heart transplantation. ESC Hear Fail. 2018;5(1):149-156.

17. Zheng J, Cai J, Li H, Zeng K, He L, Fu H, et al. Neutrophil to Lymphocyte Ratio and Platelet to Lymphocyte Ratio as Prognostic Predictors for Hepatocellular Carcinoma Patients with Various Treatments: A Meta-Analysis and Systematic Review. Cell Physiol Biochem. 2017;44(3):967-981.

18. Aydin O, Pehlivanll F. Is the Platelet to Lymphocyte Ratio a Potential Biomarker for Predicting Mortality in Peptic Ulcer Perforation? Surg Infect (Larchmt). 2019;20(4):326-331.

19. Sevencan NO, Ozkan AE. Associations between neutrophil/lymphocyte ratio, platelet/lymphocyte ratio, albuminuria and uric acid and the estimated glomerular filtration rate in hypertensive patients with chronic kidney disease stages 1-3. Arch Med Sci. 2019;15(5):1232-1239.

20. Yuan Q, Wang J, Peng Z, Zhou Q, Xiao X, Xie Y, et al. Neutrophil-to-lymphocyte ratio and incident end-stage renal disease in Chinese patients with chronic kidney disease: Results from the Chinese Cohort Study of Chronic Kidney Disease (C-STRIDE). J Transl Med. 2019 Mar 15;17(1);86.

21. Tatar E, Mirili C, Isikyakar T, Yaprak M, Guvercin G, Ozay E, et al. The association of neutrophil/lymphocyte ratio and platelet/lymphocyte ratio with clinical outcomes in geriatric patients with stage 3-5 chronic kidney disease. Acta Clin Belgica Int J Clin Lab Med. 2016;71(4):221-226.

22. Catabay C, Obi Y, Streja E, Soohoo M, Park C, Rhee CM, et al. Lymphocyte Cell Ratios and Mortality among Incident Hemodialysis Patients. Am J Nephrol. 2017;46(5):408-416.

23. Lu X, Wang S, Zhang G, Xiong R, Li H. High neutrophil-to-lymphocyte ratio is a significant predictor of cardiovascular and all-cause mortality in patients undergoing peritoneal dialysis. Kidney Blood Press Res. 2018;43(2):490-499.

24. Sato H, Takeuchi Y, Matsuda K, Kagaya S, Saito A, Fukami H, et al. Pre-Dialysis NeutrophilLymphocyte Ratio, a Novel and Strong Short-Term Predictor of All-Cause Mortality in Patients With Diabetic Nephropathy: Results From a Single-Center Study. Ther Apher Dial. 2017;21(4):370-377.

25. Neovius M, Jacobson SH, Eriksson JK, Elinder CG, Hylander B. Mortality in chronic kidney disease and renal replacement therapy: A population-based cohort study. BMJ Open. 2014;4(2).

26. Peterson K, Anderson J, Boundy E, Ferguson L, McCleery E, Waldrip K. Mortality disparities in racial/ethnic minority groups in the veterans health administration: An evidence review and Map. Am J Public Health. 2018;108(3):e1-e11. 
27. Desai N, Lora CM, Lash JP, Ricardo AC. CKD and ESRD in US Hispanics. Am J Kidney Dis. 2019;73(1):102-111.

28. Branch M, German C, Bertoni A, Yeboah J. Incremental risk of cardiovascular disease and/or chronic kidney disease for future ASCVD and mortality in patients with type 2 diabetes mellitus: ACCORD trial. J Diabetes Complications. 2019;33(7):468-472.

29. Moranne O, Fafin C, Roche S, Francois M, Allot V, Potier J, et al. Treatment plans and outcomes in elderly patients reaching advanced chronic kidney disease. Nephrol Dial Transplant. 2018;33(12):2182-2191.

30. Imig JD, Ryan MJ. Immune and inflammatory role in renal disease. Compr Physiol. 2013;3(2):957-976.

31. Okyay GU, Inal S, Onec K, Er RE, Paşaoğlu Ö, Paşaoğlu H, et al. Neutrophil to lymphocyte ratio in evaluation of inflammation in patients with chronic kidney disease. Ren Fail. 2013;35(1):29-36.

32. Toraman A, Neşe N, Özyurt B, Kürşat S. Association between neutrophil-lymphocyte \& platelet lymphocyte ratios with prognosis \& mortality in rapidly progressive glomerulonephritis. Indian J Med Res. 2019;150(4):399-406.

33. Zeng M, Liu Y, Liu F, Peng Y, Sun L, Xiao L. J-shaped association of platelet-to-lymphocyte ratio with 5 -year mortality among patients with chronic kidney disease in a prospective cohort study. Int Urol Nephrol. 2020;52(10):1943-1957.

34. Cognasse F, Laradi S, Berthelot P, Bourlet T, Marotte H, Mismetti P, et al. Platelet inflammatory response to stress. Front Immunol. 2019;10(JUN):1478.

35. Akbas EM, Demirtas L, Ozcicek A, Timuroglu A, Bakirci EM, Hamur H, et al. Association of epicardial adipose tissue, neutrophil-to-lymphocyte ratio and platelet-to-lymphocyte ratio with diabetic nephropathy. Int J Clin Exp Med. 2014;7(7):1794-180

\section{Hosted file}

Tables.pdf available at https://authorea.com/users/393219/articles/506895-neutrophil-tolymphocyte-ratio-and-platelet-to-lymphocyte-ratio-as-a-risk-factor-for-mortality-inperuvian-adults-with-chronic-kidney-disease

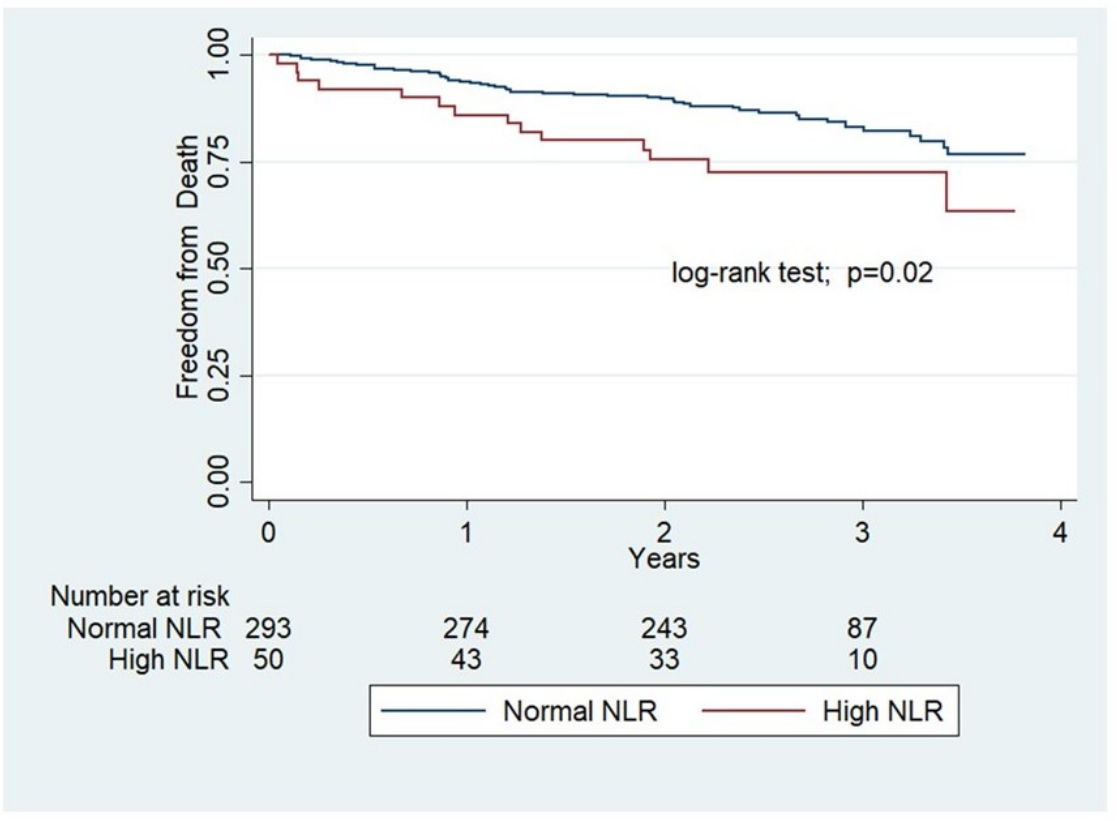




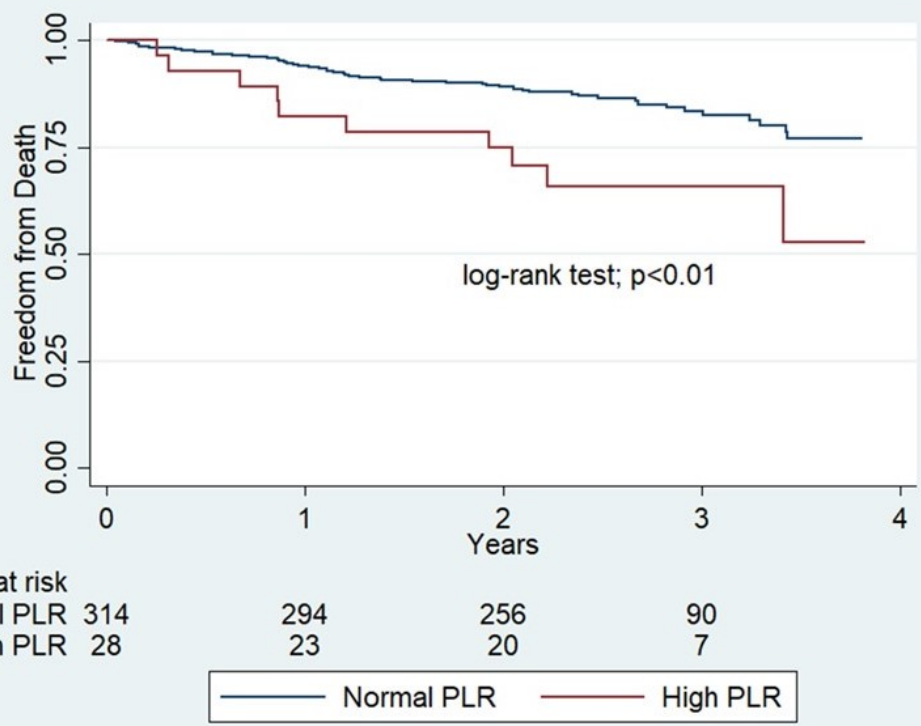

